\title{
Pet-Based Novel Imaging Techniques with Recently Introduced Radiotracers
}

Editors

MONA-ELISABETH REVHEIM

ABASS ALAVI

PET CLINICS

www.pet.theclinics.com

Consulting Editor

ABASS ALAVI

April 2021 - Volume 16 - Number 2 